\title{
Correction to "Multiconfiguration Pair-Density Functional Theory Spectral Calculations Are Stable to Adding Diffuse Basis Functions"
}

\author{
Chad E. Hoyer, Laura Gagliardi,* and Donald G. Truhlar* \\ Department of Chemistry, Chemical Theory Center, and Minnesota Supercomputing Institute, \\ University of Minnesota, Minneapolis, Minnesota 55455, USA
}

Date of preparation: November 25, 2015

\section{- TABLE OF CONTENTS}

Updated tables from manuscript of original paper

S-3 to S-4

Table 1C. Updated Table 1 S-3

Table 2C. Updated Table 2 S-4

Table 3C. Updated Table 3 S-4

Updated Supporting Information tables S-5 to S-12

Table S1C. Updated Table S1 S-5

Table S2C. Updated Table S2 S-6

Table S3C. Updated Table S3 S-7

Table S4C. Updated Table S4 S-8

Table S5C. Updated Table S5 S-9

Table S6C. Updated Table S6 S-9

Table S7C. Updated Table S7 S-10

Table S8C. Updated Table S8 
Table S9C. Updated Table S9

Table S10C. Updated Table S10

S-12

Table S11C. Updated Table S11 


\section{- UPDATED TABLES}

Table 1C. Excitation energies (in eV) of atoms. The def2-QZVP basis set was used for the valence excitations ( $\mathrm{Li}, \mathrm{Be}, \mathrm{Na}, \mathrm{Mg}, \mathrm{K}$ ), and aug-cc-pVQZ was used for Rydberg excitations (the other atoms).

\begin{tabular}{|l|c|c|c|c|c|c|c|c|}
\hline & states & type & CASSCF & CASPT2 & tPBE $^{a}$ & PBE $^{b}$ & PBE0 $^{b}$ & exp $^{\text {S1 }}$ \\
\hline $\mathrm{H}$ & ${ }^{2} \mathrm{~S}-{ }^{2} \mathrm{~S}$ & $1 s-2 s$ & 10.22 & 10.22 & 9.88 & 8.05 & 8.67 & 10.20 \\
\hline $\mathrm{He}$ & ${ }^{1} \mathrm{~S}-{ }^{1} \mathrm{~S}$ & $1 s^{2}-1 s 2 s$ & 20.15 & 20.72 & 20.42 & 17.61 & 18.75 & 20.62 \\
\hline $\mathrm{Li}$ & ${ }^{2} \mathrm{~S}-{ }^{2} \mathrm{P}$ & $2 s-2 p$ & 1.84 & 1.84 & 1.60 & 2.00 & 1.97 & 1.85 \\
\hline $\mathrm{Be}$ & ${ }^{1} \mathrm{~S}-{ }^{1} \mathrm{P}$ & $2 s^{2}-2 s 2 p$ & 5.59 & 5.42 & 3.82 & 4.99 & 4.99 & 5.28 \\
\hline $\mathrm{B}$ & ${ }^{2} \mathrm{P}-{ }^{2} \mathrm{~S}$ & $2 p-3 s$ & 4.51 & 4.93 & 5.05 & 4.14 & 4.56 & 4.96 \\
\hline $\mathrm{C}$ & ${ }^{3} \mathrm{P}-{ }^{3} \mathrm{P}$ & $2 p^{2}-2 p 3 s$ & 6.88 & 7.52 & 7.55 & 6.29 & 6.90 & 7.48 \\
\hline $\mathrm{N}$ & ${ }^{4} \mathrm{~S}-{ }^{4} \mathrm{P}$ & $2 p^{3}-2 p^{2} 3 s$ & 9.31 & 10.49 & 9.84 & 8.76 & 9.57 & 10.33 \\
\hline $\mathrm{O}$ & ${ }^{3} \mathrm{P}-{ }^{3} \mathrm{~S}$ & $2 p^{4}-2 p^{3} 3 s$ & 10.07 & 9.79 & 10.93 & 8.97 & 9.62 & 9.51 \\
\hline $\mathrm{F}$ & ${ }^{2} \mathrm{P}-{ }^{2} \mathrm{P}$ & $2 p^{5}-2 p^{4} 3 s$ & 12.74 & 13.66 & 13.68 & 12.66 & 13.68 & 12.98 \\
\hline $\mathrm{Ne}$ & ${ }^{1} \mathrm{~S}-{ }^{1} \mathrm{P}$ & $2 p^{6}-2 p^{5} 3 s$ & 15.38 & 18.05 & 16.28 & 15.85 & 17.02 & 16.71 \\
\hline $\mathrm{Na}$ & ${ }^{2} \mathrm{~S}-{ }^{2} \mathrm{P}$ & $3 s-3 p$ & 1.97 & 2.05 & 1.94 & 2.13 & 2.09 & 2.10 \\
\hline $\mathrm{Mg}$ & ${ }^{1} \mathrm{~S}-{ }^{1} \mathrm{P}$ & $3 s^{2}-3 s 3 p$ & 4.04 & 4.41 & 3.64 & 4.24 & 4.25 & 4.35 \\
\hline $\mathrm{Al}$ & ${ }^{2} \mathrm{P}-{ }^{2} \mathrm{~S}$ & $3 p-4 s$ & 2.71 & 3.08 & 3.03 & 2.73 & 2.96 & 3.14 \\
\hline $\mathrm{Si}$ & ${ }^{3} \mathrm{P}-{ }^{3} \mathrm{P}$ & $3 p^{2}-3 p 4 s$ & 4.48 & 4.93 & 4.82 & 4.29 & 4.62 & 4.92 \\
\hline $\mathrm{P}$ & ${ }^{4} \mathrm{~S}-{ }^{4} \mathrm{P}$ & $3 p^{3}-3 p^{2} 4 s$ & 6.40 & 7.04 & 6.74 & 6.07 & 6.52 & 6.96 \\
\hline $\mathrm{S}$ & ${ }^{3} \mathrm{P}-{ }^{3} \mathrm{~S}$ & $3 p^{4}-3 p^{3} 4 s$ & 6.75 & 7.00 & 7.48 & 6.85 & 7.30 & 6.82 \\
\hline $\mathrm{Cl}$ & ${ }^{2} \mathrm{P}-{ }^{2} \mathrm{P}$ & $3 p^{5}-3 p^{4} 4 s$ & 8.95 & 9.52 & 9.59 & 8.86 & 9.41 & 9.19 \\
\hline $\mathrm{Ar}$ & ${ }^{1} \mathrm{~S}-{ }^{1} \mathrm{P}$ & $3 p^{6}-3 p^{5} 4 s$ & 11.25 & 12.29 & 11.77 & 11.04 & 11.70 & 11.68 \\
\hline $\mathrm{K}$ & ${ }^{2} \mathrm{~S}-{ }^{2} \mathrm{P}$ & $4 s-4 p$ & 1.40 & 1.57 & 1.43 & 1.57 & 1.53 & 1.61 \\
\hline & & & & & & & & \\
\hline $\mathrm{MSE}$ & & & -0.32 & 0.20 & -0.06 & -0.71 & -0.24 & \\
\hline $\mathrm{MUE}$ & & & 0.41 & 0.22 & 0.42 & 0.74 & 0.45 & \\
\hline $\mathrm{SD}$ & & & 0.42 & 0.35 & 0.60 & 0.80 & 0.63 & \\
\hline
\end{tabular}

${ }^{a} \mathrm{MC}-\mathrm{PDFT}$

${ }^{b}$ TD-KS-DFT

${ }^{c}$ average value obtained from the different calculations

${ }^{d}$ mean signed error with respect to experiment

${ }^{e}$ mean unsigned error with respect to experiment

${ }^{f}$ standard deviation of the signed errors from their mean 
Table 2C. Rydberg excitation energies (in eV) of first- and second-row atoms upon doubling the number of diffuse basis functions.

\begin{tabular}{|c|c|c|c|c|c|c|c|c|}
\hline & states & basis set & CASSCF & CASPT2 & $\mathrm{tPBE}^{a}$ & $\mathrm{PBE}^{b}$ & $\mathrm{PBE}^{b}$ & $\exp ^{\mathrm{S} 1}$ \\
\hline $\mathrm{H}$ & ${ }^{2} \mathrm{~S}-{ }^{2} \mathrm{~S}$ & $\begin{array}{c}\text { aug-cc-pVQZ } \\
\text { d-aug-cc-pVQZ }\end{array}$ & $\begin{array}{l}10.22 \\
10.21\end{array}$ & $\begin{array}{l}10.22 \\
10.21\end{array}$ & $\begin{array}{l}9.88 \\
9.89\end{array}$ & $\begin{array}{l}8.05 \\
7.64\end{array}$ & $\begin{array}{l}8.67 \\
8.44\end{array}$ & 10.20 \\
\hline $\mathrm{He}$ & ${ }^{1} \mathrm{~S}-{ }^{\mathrm{I}} \mathrm{S}$ & $\begin{array}{c}\text { aug-cc-pVQZ } \\
\text { d-aug-cc-pVQZ }\end{array}$ & $\begin{array}{l}20.15 \\
19.88\end{array}$ & $\begin{array}{l}20.72 \\
20.46\end{array}$ & $\begin{array}{l}20.42 \\
20.30\end{array}$ & $\begin{array}{l}17.61 \\
16.21\end{array}$ & $\begin{array}{l}18.75 \\
17.70\end{array}$ & 20.62 \\
\hline B & ${ }^{2} \mathrm{P}-{ }^{2} \mathrm{~S}$ & $\begin{array}{c}\text { aug-cc-pVQZ } \\
\text { d-aug-cc-pVQZ }\end{array}$ & $\begin{array}{l}4.51 \\
4.45\end{array}$ & $\begin{array}{l}4.93 \\
4.88\end{array}$ & $\begin{array}{l}5.05 \\
4.99\end{array}$ & $\begin{array}{l}4.14 \\
3.85 \\
\end{array}$ & $\begin{array}{l}4.56 \\
4.37 \\
\end{array}$ & 4.96 \\
\hline $\mathrm{C}$ & ${ }^{3} \mathrm{P}-{ }^{3} \mathrm{P}$ & $\begin{array}{c}\text { aug-cc-pVQZ } \\
\text { d-aug-cc-pVQZ }\end{array}$ & $\begin{array}{l}6.88 \\
6.68 \\
\end{array}$ & $\begin{array}{l}7.52 \\
7.35\end{array}$ & $\begin{array}{l}7.55 \\
7.38 \\
\end{array}$ & $\begin{array}{l}6.29 \\
5.77 \\
\end{array}$ & $\begin{array}{l}6.90 \\
6.52 \\
\end{array}$ & 7.48 \\
\hline $\mathrm{N}$ & ${ }^{4} \mathrm{~S}-{ }^{4} \mathrm{P}$ & $\begin{array}{c}\text { aug-cc-pVQZ } \\
\text { d-aug-cc-pVQZ }\end{array}$ & $\begin{array}{l}9.31 \\
8.85\end{array}$ & $\begin{array}{l}10.49 \\
10.23\end{array}$ & $\begin{array}{l}9.84 \\
9.52\end{array}$ & $\begin{array}{l}8.76 \\
8.00 \\
\end{array}$ & $\begin{array}{l}9.57 \\
9.00 \\
\end{array}$ & 10.33 \\
\hline $\mathrm{O}$ & ${ }^{3} \mathrm{P}-{ }^{3} \mathrm{~S}$ & $\begin{array}{c}\text { aug-cc-pVQZ } \\
\text { d-aug-cc-pVQZ }\end{array}$ & $\begin{array}{c}10.07 \\
9.30 \\
\end{array}$ & $\begin{array}{l}9.79 \\
9.05\end{array}$ & $\begin{array}{l}10.93 \\
10.45 \\
\end{array}$ & $\begin{array}{l}8.97 \\
7.59\end{array}$ & $\begin{array}{l}9.62 \\
8.48\end{array}$ & 9.51 \\
\hline $\mathrm{F}$ & ${ }^{2} \mathrm{P}-{ }^{2} \mathrm{P}$ & $\begin{array}{c}\text { aug-cc-pVQZ } \\
\text { d-aug-cc-pVQZ }\end{array}$ & $\begin{array}{l}12.74 \\
11.59 \\
\end{array}$ & $\begin{array}{l}13.66 \\
12.64 \\
\end{array}$ & $\begin{array}{l}13.68 \\
12.77 \\
\end{array}$ & $\begin{array}{l}12.66 \\
10.31 \\
\end{array}$ & $\begin{array}{l}13.68 \\
11.48 \\
\end{array}$ & 12.98 \\
\hline $\mathrm{Ne}$ & ${ }^{1} \mathrm{~S}-{ }^{\mathrm{I}} \mathrm{P}$ & $\begin{array}{c}\text { aug-cc-pVQZ } \\
\text { d-aug-cc-pVQZ }\end{array}$ & $\begin{array}{l}15.38 \\
13.68 \\
\end{array}$ & $\begin{array}{l}18.05 \\
16.63\end{array}$ & $\begin{array}{l}16.28 \\
16.25 \\
\end{array}$ & $\begin{array}{l}15.85 \\
13.53\end{array}$ & $\begin{array}{l}17.02 \\
15.01 \\
\end{array}$ & 16.71 \\
\hline $\mathrm{MSE}^{c}$ & & $\begin{array}{c}\text { aug-cc-pVQZ } \\
\text { d-aug-cc-pVQZ }\end{array}$ & $\begin{array}{l}-0.44 \\
-1.02 \\
\end{array}$ & $\begin{array}{c}0.32 \\
-0.17 \\
\end{array}$ & $\begin{array}{c}0.11 \\
-0.15\end{array}$ & $\begin{array}{l}-1.31 \\
-2.49 \\
\end{array}$ & $\begin{array}{l}-0.50 \\
-1.47\end{array}$ & \\
\hline $\mathrm{MUE}^{d}$ & & $\begin{array}{c}\text { aug-cc-pVQZ } \\
\text { d-aug-cc-pVQZ }\end{array}$ & $\begin{array}{l}0.59 \\
1.02 \\
\end{array}$ & $\begin{array}{l}0.33 \\
0.17 \\
\end{array}$ & $\begin{array}{l}0.46 \\
0.40\end{array}$ & $\begin{array}{l}1.31 \\
2.49\end{array}$ & $\begin{array}{l}0.78 \\
1.47 \\
\end{array}$ & \\
\hline
\end{tabular}

\footnotetext{
${ }^{a} \mathrm{MC}-\mathrm{PDFT}$

${ }^{b}$ TD-KS-DFT

${ }^{c}$ mean signed error

${ }^{d}$ mean unsigned error
}

Table 3C. Mean unsigned errors in Rydberg excitation energies (in eV) of first- and second-row atoms upon doubling the number of diffuse basis functions.

\begin{tabular}{cccccc}
\hline basis set & CASSCF & CASPT2 $^{\text {tPBE }}$ & PBE $^{b}$ & PBE0 $^{b}$ \\
\hline aug-cc-pVTZ & 0.49 & 0.42 & 0.48 & 1.07 & 0.85 \\
aug-cc-pVQZ & 0.59 & 0.33 & 0.46 & 1.31 & 0.78 \\
aug-cc-pV5Z & 0.68 & 0.24 & 0.48 & 1.51 & 0.78 \\
& & & & & \\
d-aug-cc-pVTZ & 1.01 & 0.24 & 0.57 & 2.48 & 1.46 \\
d-aug-cc-pVQZ & 1.02 & 0.17 & 0.40 & 2.49 & 1.47 \\
d-aug-cc-pV5Z & 1.02 & 0.13 & 0.53 & 2.50 & 1.48 \\
${ }^{a}$ MC-PDFT & & & & & \\
${ }^{b}$ TD-KS-DFT & & & & &
\end{tabular}


Table S1C. Valence excitation energies (in $\mathrm{eV}$ ) of selected atoms (Be, $\mathrm{Na}$, and $\mathrm{Mg}$ ) are reported.

\begin{tabular}{|c|c|c|c|c|c|c|c|c|}
\hline & \multirow{2}{*}{ states } & basis set & CASSCF & CASPT2 $^{*}$ tPBE $^{a}$ & PBE $^{b}$ & PBE0 $^{b}$ & $\exp ^{\text {S1 }^{2}}$ \\
\hline $\mathrm{Be}$ & ${ }^{1} \mathrm{~S}-{ }^{1} \mathrm{P}$ & def2-QZVP & 5.59 & 5.42 & 3.82 & 4.99 & 4.99 & 5.28 \\
& & aug-cc-pVQZ & 5.50 & 5.35 & 3.86 & 4.90 & 4.94 & \\
\hline $\mathrm{Na}$ & ${ }^{2} \mathrm{~S}-{ }^{2} \mathrm{P}$ & def2-QZVP & 1.97 & 2.05 & 1.94 & 2.13 & 2.09 & 2.10 \\
& & aug-cc-pVQZ & 1.97 & 2.01 & 1.94 & 2.13 & 2.09 & \\
\hline $\mathrm{Mg}$ & \multirow{2}{*}{$\mathrm{S}-{ }^{1} \mathrm{P}$} & def2-QZVP & 4.04 & 4.41 & 3.64 & 4.24 & 4.25 & 4.35 \\
& & aug-cc-pVQZ & 3.93 & 4.31 & 3.73 & 4.18 & 4.21 & \\
\hline & & & & & & & & \\
\hline $\mathrm{MSE}^{c}$ & & def2-QZVP & -0.04 & 0.05 & -0.77 & -0.12 & -0.13 & \\
& & aug-cc-pVQZ & -0.11 & -0.02 & -0.73 & -0.17 & -0.16 & \\
\hline $\mathrm{MUE}^{d}$ & & def2-QZVP & 0.25 & 0.08 & 0.77 & 0.14 & 0.13 & \\
& & aug-cc-pVQZ & 0.25 & 0.07 & 0.73 & 0.19 & 0.16 & \\
\hline
\end{tabular}

${ }^{a} \mathrm{MC}-\mathrm{PDFT}$

${ }^{b}$ TD-KS-DFT

${ }^{c}$ mean signed error

${ }^{d}$ mean unsigned error 
Table S2C. Rydberg excitation energies (in eV) of first- and second-row atoms upon doubling the number of diffuse basis functions.

\begin{tabular}{|c|c|c|c|c|c|c|c|c|}
\hline & states & basis set & CASSCF & CASPT2 & $\mathrm{tPBE}^{a}$ & $\mathrm{PBE}^{b}$ & $\mathrm{PBE} 0^{b}$ & $\exp ^{\mathrm{S} 1}$ \\
\hline $\mathrm{H}$ & ${ }^{2} \mathrm{~S}-{ }^{2} \mathrm{~S}$ & $\begin{array}{c}\text { aug-cc-pVTZ } \\
\text { d-aug-cc-pVTZ }\end{array}$ & $\begin{array}{l}10.23 \\
10.20\end{array}$ & $\begin{array}{l}10.23 \\
10.20\end{array}$ & $\begin{array}{l}9.88 \\
9.89\end{array}$ & $\begin{array}{l}8.14 \\
7.64\end{array}$ & $\begin{array}{l}8.74 \\
8.44\end{array}$ & 10.20 \\
\hline $\mathrm{He}$ & ${ }^{\mathrm{T}} \mathrm{S}-{ }^{\mathrm{I}} \mathrm{S}$ & $\begin{array}{c}\text { aug-cc-pVTZ } \\
\text { d-aug-cc-pVTZ }\end{array}$ & $\begin{array}{l}20.25 \\
19.90\end{array}$ & $\begin{array}{l}20.77 \\
20.43\end{array}$ & $\begin{array}{l}20.48 \\
20.32\end{array}$ & $\begin{array}{l}17.86 \\
16.23\end{array}$ & $\begin{array}{l}18.96 \\
17.72\end{array}$ & 20.62 \\
\hline B & ${ }^{2} \mathrm{P}-{ }^{2} \mathrm{~S}$ & $\begin{array}{c}\text { aug-cc-pVTZ } \\
\text { d-aug-cc-pVTZ }\end{array}$ & $\begin{array}{l}4.54 \\
4.45\end{array}$ & $\begin{array}{l}4.92 \\
4.84\end{array}$ & $\begin{array}{l}5.07 \\
5.04\end{array}$ & $\begin{array}{l}4.23 \\
3.86\end{array}$ & $\begin{array}{l}4.62 \\
4.38\end{array}$ & 4.96 \\
\hline $\mathrm{C}$ & ${ }^{3} \mathrm{P}-{ }^{3} \mathrm{P}$ & $\begin{array}{c}\text { aug-cc-pVTZ } \\
\text { d-aug-cc-pVTZ }\end{array}$ & $\begin{array}{l}6.96 \\
6.68\end{array}$ & $\begin{array}{l}7.53 \\
7.31\end{array}$ & $\begin{array}{l}7.56 \\
7.38\end{array}$ & $\begin{array}{l}6.43 \\
5.78\end{array}$ & $\begin{array}{l}7.01 \\
6.52\end{array}$ & 7.48 \\
\hline $\mathrm{N}$ & ${ }^{4} \mathrm{~S}-{ }^{4} \mathrm{P}$ & $\begin{array}{c}\text { aug-cc-pVTZ } \\
\text { d-aug-cc-pVTZ }\end{array}$ & $\begin{array}{l}9.45 \\
8.85\end{array}$ & $\begin{array}{l}10.51 \\
10.18\end{array}$ & $\begin{array}{l}9.93 \\
9.51\end{array}$ & $\begin{array}{l}8.94 \\
7.90\end{array}$ & $\begin{array}{l}9.71 \\
9.01\end{array}$ & 10.33 \\
\hline $\mathrm{O}$ & ${ }^{3} \mathrm{P}-{ }^{3} \mathrm{~S}$ & $\begin{array}{c}\text { aug-cc-pVTZ } \\
\text { d-aug-cc-pVTZ }\end{array}$ & $\begin{array}{c}10.31 \\
9.31\end{array}$ & $\begin{array}{l}9.90 \\
8.92\end{array}$ & $\begin{array}{l}11.15 \\
10.46\end{array}$ & $\begin{array}{l}9.27 \\
7.60\end{array}$ & $\begin{array}{l}9.88 \\
8.49\end{array}$ & 9.51 \\
\hline $\mathrm{F}$ & ${ }^{2} \mathrm{P}-{ }^{2} \mathrm{P}$ & $\begin{array}{c}\text { aug-cc-pVTZ } \\
\text { d-aug-cc-pVTZ }\end{array}$ & $\begin{array}{l}13.10 \\
11.61\end{array}$ & $\begin{array}{l}13.85 \\
12.51\end{array}$ & $\begin{array}{l}14.12 \\
12.85\end{array}$ & $\begin{array}{l}13.06 \\
10.34\end{array}$ & $\begin{array}{l}14.04 \\
11.51\end{array}$ & 12.98 \\
\hline $\mathrm{Ne}$ & ${ }^{1} \mathrm{~S}-{ }^{\mathrm{I}} \mathrm{P}$ & $\begin{array}{c}\text { aug-cc-pVTZ } \\
\text { d-aug-cc-pVTZ }\end{array}$ & $\begin{array}{l}15.89 \\
13.70\end{array}$ & $\begin{array}{l}18.35 \\
16.51\end{array}$ & $\begin{array}{l}16.69 \\
14.84\end{array}$ & $\begin{array}{l}16.43 \\
13.58\end{array}$ & $\begin{array}{l}17.53 \\
15.05\end{array}$ & 16.71 \\
\hline $\mathrm{MSE}^{c}$ & & $\begin{array}{c}\text { aug-cc-pVTZ } \\
\text { d-aug-cc-pVTZ }\end{array}$ & $\begin{array}{l}-0.26 \\
-1.01\end{array}$ & $\begin{array}{c}0.41 \\
-0.23\end{array}$ & $\begin{array}{r}0.26 \\
-0.31\end{array}$ & $\begin{array}{l}-1.05 \\
-2.48\end{array}$ & $\begin{array}{l}-0.29 \\
-1.46\end{array}$ & \\
\hline $\mathrm{MUE}^{d}$ & & $\begin{array}{c}\text { aug-cc-pVTZ } \\
\text { d-aug-cc-pVTZ }\end{array}$ & $\begin{array}{l}0.49 \\
1.01\end{array}$ & $\begin{array}{l}0.42 \\
0.24\end{array}$ & $\begin{array}{l}0.48 \\
0.57\end{array}$ & $\begin{array}{l}1.07 \\
2.48\end{array}$ & $\begin{array}{l}0.85 \\
1.46\end{array}$ & \\
\hline
\end{tabular}

${ }^{a} \mathrm{MC}-\mathrm{PDFT}$

${ }^{b}$ TD-KS-DFT

${ }^{c}$ mean signed error

${ }^{d}$ mean unsigned error 
Table S3C. Rydberg excitation energies (in eV) of first- and second-row atoms upon doubling the number of diffuse basis functions.

\begin{tabular}{|c|c|c|c|c|c|c|c|c|}
\hline & \multirow{2}{*}{ states } & basis set & CASSCF & CASPT2 & \multirow{2}{*}{$\mathrm{tPBE}^{a}$} & $\mathrm{PBE}^{b}$ & $\mathrm{PBE0}^{b}$ & $\exp ^{\mathrm{S} 1}$ \\
\hline $\mathrm{H}$ & ${ }^{2} \mathrm{~S}-{ }^{2} \mathrm{~S}$ & aug-cc-pV5Z & 10.21 & 10.21 & 9.89 & 7.93 & 8.59 & 10.20 \\
& & d-aug-cc-pV5Z & 10.21 & 10.21 & 9.89 & 7.63 & 8.44 & \\
\hline $\mathrm{He}$ & ${ }^{\mathrm{S}} \mathrm{S}-{ }^{1} \mathrm{~S}$ & aug-cc-pV5Z & 20.10 & 20.69 & 20.40 & 17.46 & 18.63 & 20.62 \\
& & d-aug-cc-pV5Z & 19.87 & 20.48 & 20.30 & 16.19 & 17.70 & \\
\hline $\mathrm{B}$ & ${ }^{2} \mathrm{P}-{ }^{2} \mathrm{~S}$ & aug-cc-pV5Z & 4.49 & 4.94 & 5.03 & 4.10 & 4.53 & 4.96 \\
& & d-aug-cc-pV5Z & 4.45 & 4.90 & 4.99 & 3.86 & 4.37 & \\
\hline $\mathrm{C}$ & ${ }^{3} \mathrm{P}-{ }^{3} \mathrm{P}$ & aug-cc-pV5Z & 6.84 & 7.50 & 7.44 & 6.20 & 6.83 & 7.48 \\
& & d-aug-cc-pV5Z & 6.68 & 7.38 & 7.44 & 5.77 & 6.52 & \\
\hline $\mathrm{N}$ & ${ }^{4} \mathrm{~S}-{ }^{4} \mathrm{P}$ & aug-cc-pV5Z & 9.22 & 10.45 & 9.75 & 8.62 & 9.46 & 10.33 \\
& & d-aug-cc-pV5Z & 8.85 & 10.26 & 9.50 & 7.99 & 8.99 & \\
\hline $\mathrm{O}$ & ${ }^{3} \mathrm{P}-{ }^{3} \mathrm{~S}$ & aug-cc-pV5Z & 9.89 & 9.68 & 10.80 & 8.73 & 9.41 & 9.51 \\
& & d-aug-cc-pV5Z & 9.30 & 9.11 & 11.32 & 7.57 & 8.47 & \\
\hline $\mathrm{F}$ & ${ }^{2} \mathrm{P}-{ }^{2} \mathrm{P}$ & aug-cc-pV5Z & 12.49 & 13.49 & 13.44 & 12.33 & 13.40 & 12.98 \\
& & d-aug-cc-pV5Z & 11.59 & 12.70 & 12.77 & 10.28 & 11.46 & \\
\hline $\mathrm{Ne}$ & ${ }^{1} \mathrm{~S}-{ }^{1} \mathrm{P}$ & aug-cc-pV5Z & 14.93 & 17.72 & 15.87 & 15.30 & 16.53 & 16.71 \\
& & d-aug-cc-pV5Z & 13.67 & 16.70 & 16.02 & 13.47 & 14.96 & \\
\hline & & & & & & & & \\
\hline $\mathrm{MSE}^{c}$ & & aug-cc-pV5Z & -0.58 & 0.24 & -0.02 & -1.51 & -0.68 & \\
& & d-aug-cc-pV5Z & -1.02 & -0.13 & -0.07 & -2.50 & -1.48 & \\
\hline $\mathrm{MUE}^{d}$ & & aug-cc-pV5Z & 0.68 & 0.24 & 0.48 & 1.51 & 0.78 & \\
& & d-aug-cc-pV5Z & 1.02 & 0.13 & 0.53 & 2.50 & 1.48 & \\
\hline
\end{tabular}

${ }^{a} \mathrm{MC}-\mathrm{PDFT}$

${ }^{b}$ TD-KS-DFT

${ }^{c}$ mean signed error

${ }^{d}$ mean unsigned error 
Table S4C. Total energies (in atomic units) for all states and $\left\langle\mathrm{S}^{2}>\right.$ values with the aug-cc-pVTZ basis set for Rydberg excitations of atoms $\mathrm{H}$ to $\mathrm{Ne}$.

\begin{tabular}{|c|c|c|c|c|c|c|c|c|}
\hline & states & CASSCF & CASPT2 & tPBE $^{a}$ & PBE $^{b}$ & PBE0 $^{b}$ & $\begin{array}{c}\text { PBE } \\
<\mathrm{S}^{2}>\end{array}$ & $\begin{array}{c}\text { PBE0 } \\
<\mathrm{S}^{2}>\end{array}$ \\
\hline $\mathrm{H}$ & ${ }^{2} \mathrm{~S}$ & -0.49982 & -0.49982 & -0.49926 & -0.49980 & -0.50115 & 0.8 & 0.8 \\
& ${ }^{2} \mathrm{~S}$ & -0.12399 & -0.12399 & -0.13618 & -0.20067 & -0.18007 & 0.8 & 0.8 \\
\hline $\mathrm{He}$ & ${ }^{1} \mathrm{~S}$ & -2.85302 & -2.89288 & -2.88200 & -2.89242 & -2.89468 & 0.0 & 0.0 \\
& ${ }^{1} \mathrm{~S}$ & -2.10883 & -2.12953 & -2.12945 & -2.23594 & -2.19804 & 0.0 & 0.0 \\
\hline $\mathrm{B}$ & ${ }^{2} \mathrm{P}$ & -24.55903 & -24.59089 & -24.60844 & -24.61007 & -24.61818 & 0.8 & 0.8 \\
& ${ }^{2} \mathrm{~S}$ & -24.39217 & -24.41018 & -24.42194 & -24.45463 & -24.44823 & 0.8 & 0.8 \\
\hline $\mathrm{C}$ & ${ }^{3} \mathrm{P}$ & -37.69917 & -37.76893 & -37.78346 & -37.79562 & -37.80486 & 2.0 & 2.0 \\
& ${ }^{3} \mathrm{P}$ & -37.44339 & -37.49223 & -37.50567 & -37.55926 & -37.54719 & 2.0 & 2.0 \\
\hline $\mathrm{N}$ & ${ }^{4} \mathrm{~S}$ & -54.37897 & -54.50173 & -54.49908 & -54.53118 & -54.54257 & 3.8 & 3.8 \\
& ${ }^{4} \mathrm{P}$ & -54.03164 & -54.11542 & -54.13429 & -54.20257 & -54.18564 & 3.8 & 3.8 \\
\hline $\mathrm{O}$ & ${ }^{3} \mathrm{P}$ & -74.80040 & -74.96124 & -74.98302 & -75.00778 & -75.01627 & 2.0 & 2.0 \\
& ${ }^{3} \mathrm{~S}$ & -74.42150 & -74.59742 & -74.57311 & -74.66713 & -74.65310 & 3.5 & 3.4 \\
\hline $\mathrm{F}$ & ${ }^{2} \mathrm{P}$ & -99.37891 & -99.60904 & -99.62568 & -99.66540 & -99.67183 & 0.8 & 0.8 \\
& ${ }^{2} \mathrm{P}$ & -98.89760 & -99.10008 & -99.10693 & -99.18528 & -99.15599 & 1.1 & 1.1 \\
\hline $\mathrm{Ne}$ & ${ }^{1} \mathrm{~S}$ & -128.47373 & -128.79692 & -128.77434 & -128.85134 & -128.85724 & 0.0 & 0.0 \\
& ${ }^{1} \mathrm{P}$ & -127.88960 & -128.12252 & -128.16083 & -128.24771 & -128.21293 & 0.0 & 0.0 \\
\hline
\end{tabular}

${ }^{a} \mathrm{MC}-\mathrm{PDFT}$

${ }^{b}$ TD-KS-DFT 
Table S5C. Total energies (in atomic units) for all states and $<\mathrm{S}^{2}>$ values with the d-aug-ccpVTZ basis set for Rydberg excitations of atoms $\mathrm{H}$ to Ne.

\begin{tabular}{|c|c|c|c|c|c|c|c|c|}
\hline & states & CASSCF & CASPT2 & tPBE $^{a}$ & $\mathrm{PBE}^{b}$ & $\mathrm{PBE0}^{b}$ & $\begin{array}{c}\mathrm{PBE} \\
<\mathrm{S}^{2}>\end{array}$ & $\begin{array}{c}\text { PBE0 } \\
<\mathrm{S}^{2}>\end{array}$ \\
\hline $\mathrm{H}$ & ${ }^{2} \mathrm{~S}$ & -0.49982 & -0.49982 & -0.49926 & -0.49981 & -0.50115 & 0.8 & 0.8 \\
& ${ }^{2} \mathrm{~S}$ & -0.12482 & -0.12482 & -0.13597 & -0.21917 & -0.19100 & 0.8 & 0.8 \\
\hline $\mathrm{He}$ & ${ }^{\mathrm{S}} \mathrm{S}$ & -2.85176 & -2.89254 & -2.88044 & -2.89243 & -2.89469 & 0.0 & 0.0 \\
& ${ }^{1} \mathrm{~S}$ & -2.12048 & -2.14182 & -2.13380 & -2.29589 & -2.24360 & 0.0 & 0.0 \\
\hline $\mathrm{B}$ & ${ }^{2} \mathrm{P}$ & -24.55888 & -24.59098 & -24.60852 & -24.61008 & -24.61819 & 0.8 & 0.8 \\
& ${ }^{2} \mathrm{~S}$ & -24.39519 & -24.41294 & -24.42337 & -24.46825 & -24.45741 & 0.8 & 0.8 \\
\hline $\mathrm{C}$ & ${ }^{3} \mathrm{P}$ & -37.69800 & -37.76908 & -37.78293 & -37.79563 & -37.80487 & 2.0 & 2.0 \\
& ${ }^{3} \mathrm{P}$ & -37.45240 & -37.50043 & -37.51159 & -37.58340 & -37.56510 & 2.0 & 2.0 \\
\hline $\mathrm{N}$ & ${ }^{4} \mathrm{~S}$ & -54.37318 & -54.50254 & -54.49464 & -54.53119 & -54.54257 & 3.8 & 3.8 \\
& ${ }^{4} \mathrm{P}$ & -54.04783 & -54.12830 & -54.14518 & -54.24076 & -54.21161 & 3.8 & 3.8 \\
\hline $\mathrm{O}$ & ${ }^{3} \mathrm{P}$ & -74.79942 & -74.96120 & -74.98332 & -75.00780 & -75.01628 & 2.0 & 2.0 \\
& ${ }^{3} \mathrm{~S}$ & -74.45725 & -74.63322 & -74.59880 & -74.72835 & -74.70412 & 3.2 & 3.2 \\
\hline $\mathrm{F}$ & ${ }^{2} \mathrm{P}$ & -99.37608 & -99.60886 & -99.61860 & -99.66543 & -99.67186 & 0.8 & 0.8 \\
& ${ }^{2} \mathrm{P}$ & -98.94942 & -99.14912 & -99.14654 & -99.28557 & -99.24903 & 2.0 & 2.0 \\
\hline $\mathrm{Ne}$ & ${ }^{1} \mathrm{~S}$ & -128.46584 & -128.79675 & -128.76287 & -128.85134 & -128.85725 & 0.0 & 0.0 \\
& ${ }^{1} \mathrm{P}$ & -127.96233 & -128.19007 & -128.21737 & -128.35225 & -128.30414 & 0.0 & 0.0 \\
\hline
\end{tabular}

${ }^{a} \mathrm{MC}-\mathrm{PDFT}$

${ }^{b}$ TD-KS-DFT

Table S6C. Total energies (in atomic units) for all states and $\left\langle\mathrm{S}^{2}>\right.$ values with the def2-QZVP basis set for valence excitations.

\begin{tabular}{|c|c|c|c|c|c|c|c|c|}
\hline & states & CASSCF & CASPT2 & tPBE $^{a}$ & PBE $^{b}$ & PBE0 $^{b}$ & $\begin{array}{c}\text { PBE } \\
<\mathrm{S}^{2}>\end{array}$ & $\begin{array}{c}\text { PBE0 } \\
<\mathrm{S}^{2}>\end{array}$ \\
\hline $\mathrm{Li}$ & ${ }^{2} \mathrm{~S}$ & -7.43271 & -7.43271 & -7.46186 & -7.46198 & -7.46709 & 0.8 & 0.8 \\
& ${ }^{2} \mathrm{P}$ & -7.36501 & -7.36501 & -7.40300 & -7.38847 & -7.39467 & 0.8 & 0.8 \\
\hline $\mathrm{Be}$ & ${ }^{1} \mathrm{~S}$ & -14.59329 & -14.61253 & -14.63183 & -14.62984 & -14.63656 & 0.0 & 0.0 \\
& ${ }^{1} \mathrm{P}$ & -14.38776 & -14.41352 & -14.49136 & -14.44664 & -14.45331 & 0.0 & 0.0 \\
\hline $\mathrm{Na}$ & ${ }^{2} \mathrm{~S}$ & -161.85870 & -161.96563 & -162.16563 & -162.16817 & -162.18253 & 0.8 & 0.8 \\
& ${ }^{2} \mathrm{P}$ & -161.78622 & -161.89021 & -162.09427 & -162.08989 & -162.10556 & 0.8 & 0.8 \\
\hline $\mathrm{Mg}$ & ${ }^{1} \mathrm{~S}$ & -199.61491 & -199.76585 & -199.94513 & -199.95131 & -199.96869 & 0.0 & 0.0 \\
& ${ }^{1} \mathrm{P}$ & -199.46642 & -199.60369 & -199.81130 & -199.79542 & -199.81256 & 0.0 & 0.0 \\
\hline $\mathrm{K}$ & ${ }^{2} \mathrm{~S}$ & -599.16443 & -599.30947 & -599.70124 & -599.70468 & -599.75153 & 0.8 & 0.8 \\
& ${ }^{2} \mathrm{P}$ & -599.11280 & -599.25188 & -599.64861 & -599.64687 & -599.69547 & 0.8 & 0.8 \\
\hline
\end{tabular}

${ }^{a} \mathrm{MC}-\mathrm{PDFT}$

${ }^{b}$ TD-KS-DFT 
Table S7C. Total energies (in atomic units) for all states and $<\mathrm{S}^{2}>$ values with the aug-cc-pVQZ basis set for atoms $\mathrm{H}$ to $\mathrm{Ne}$.

\begin{tabular}{|c|c|c|c|c|c|c|c|c|}
\hline & states & CASSCF & CASPT2 & tPBE $^{a}$ & PBE $^{b}$ & PBE0 $^{b}$ & $\begin{array}{c}\text { PBE } \\
<\mathrm{S}^{2}>\end{array}$ & $\begin{array}{c}\text { PBE0 } \\
<\mathrm{S}^{2}>\end{array}$ \\
\hline $\mathrm{H}$ & ${ }^{2} \mathrm{~S}$ & -0.49995 & -0.49995 & -0.49937 & -0.49993 & -0.50127 & 0.8 & 0.8 \\
& ${ }^{2} \mathrm{~S}$ & -0.12442 & -0.12442 & -0.13613 & -0.20408 & -0.18253 & 0.8 & 0.8 \\
\hline $\mathrm{He}$ & ${ }^{1} \mathrm{~S}$ & -2.85298 & -2.89539 & -2.88220 & -2.89278 & -2.89502 & 0.0 & 0.0 \\
& ${ }^{1} \mathrm{~S}$ & -2.11260 & -2.13392 & -2.13166 & -2.24561 & -2.20611 & 0.0 & 0.0 \\
\hline $\mathrm{Be}$ & ${ }^{1} \mathrm{~S}$ & -14.59115 & -14.61291 & -14.63140 & -14.62901 & -14.63613 & 0.0 & 0.0 \\
& ${ }^{1} \mathrm{P}$ & -14.38916 & -14.41646 & -14.49016 & -14.44878 & -14.45473 & 0.0 & 0.0 \\
\hline $\mathrm{B}$ & ${ }^{2} \mathrm{P}$ & -24.55982 & -24.59425 & -24.60917 & -24.61120 & -24.61920 & 0.8 & 0.8 \\
& ${ }^{2} \mathrm{~S}$ & -24.39420 & -24.41308 & -24.42343 & -24.45908 & -24.45174 & 0.8 & 0.8 \\
\hline $\mathrm{C}$ & ${ }^{3} \mathrm{P}$ & -37.70037 & -37.77539 & -37.78480 & -37.79751 & -37.80662 & 2.0 & 2.0 \\
& ${ }^{3} \mathrm{P}$ & -37.44736 & -37.49920 & -37.50724 & -37.56641 & -37.55308 & 2.0 & 2.0 \\
\hline $\mathrm{N}$ & ${ }^{4} \mathrm{~S}$ & -54.38027 & -54.51219 & -54.50066 & -54.53420 & -54.54544 & 3.8 & 3.8 \\
& ${ }^{4} \mathrm{P}$ & -54.03797 & -54.12683 & -54.13888 & -54.21223 & -54.19367 & 3.8 & 3.8 \\
\hline $\mathrm{O}$ & ${ }^{3} \mathrm{P}$ & -74.80463 & -74.97926 & -74.98614 & -75.01284 & -75.02116 & 2.0 & 2.0 \\
& ${ }^{3} \mathrm{~S}$ & -74.43460 & -74.61950 & -74.58433 & -74.68310 & -74.66754 & 3.5 & 3.4 \\
\hline $\mathrm{F}$ & ${ }^{2} \mathrm{P}$ & -99.38523 & -99.63592 & -99.62668 & -99.67310 & -99.67934 & 0.8 & 0.8 \\
& ${ }^{2} \mathrm{P}$ & -98.91691 & -99.13394 & -99.12395 & -99.20798 & -99.17666 & 1.1 & 1.2 \\
\hline $\mathrm{Ne}$ & ${ }^{1} \mathrm{~S}$ & -128.48231 & -128.83353 & -128.78179 & -128.86233 & -128.86803 & 0.0 & 0.0 \\
& ${ }^{1} \mathrm{P}$ & -127.91711 & -128.17036 & -128.18341 & -128.27969 & -128.24262 & 0.0 & 0.0 \\
\hline
\end{tabular}

${ }^{a} \mathrm{MC}-\mathrm{PDFT}$

${ }^{b}$ TD-KS-DFT 
Table S8C. Total energies (in atomic units) for all states and $<\mathrm{S}^{2}>$ values with the aug-cc-pVQZ basis set for atoms $\mathrm{Na}$ to Ar.

\begin{tabular}{|c|c|c|c|c|c|c|c|c|}
\hline & states & CASSCF & CASPT2 & tPBE $^{a}$ & PBE $^{b}$ & PBE0 $^{b}$ & $\begin{array}{c}\text { PBE } \\
<\mathrm{S}^{2}>\end{array}$ & $\begin{array}{c}\text { PBE0 } \\
<\mathrm{S}^{2}>\end{array}$ \\
\hline $\mathrm{Na}$ & ${ }^{2} \mathrm{~S}$ & -161.85863 & -161.86417 & -162.16549 & -162.16821 & -162.18243 & 0.8 & 0.8 \\
& ${ }^{2} \mathrm{P}$ & -161.78616 & -161.79042 & -162.09409 & -162.08997 & -162.10548 & 0.8 & 0.8 \\
\hline $\mathrm{Mg}$ & ${ }^{1} \mathrm{~S}$ & -199.61276 & -199.65964 & -199.94454 & -199.95007 & -199.96787 & 0.0 & 0.0 \\
& ${ }^{1} \mathrm{P}$ & -199.46832 & -199.50134 & -199.80759 & -199.79663 & -199.81319 & 0.0 & 0.0 \\
\hline $\mathrm{Al}$ & ${ }^{2} \mathrm{P}$ & -241.89420 & -241.94288 & -242.22298 & -242.23328 & -242.25478 & 0.8 & 0.8 \\
& ${ }^{2} \mathrm{~S}$ & -241.79455 & -241.82980 & -242.11197 & -242.13280 & -242.14614 & 0.8 & 0.8 \\
\hline $\mathrm{Si}$ & ${ }^{3} \mathrm{P}$ & -288.86191 & -288.95176 & -289.21707 & -289.22968 & -289.25658 & 2.0 & 2.0 \\
& ${ }^{3} \mathrm{P}$ & -288.69728 & -288.77076 & -289.03995 & -289.07196 & -289.08680 & 2.0 & 2.0 \\
\hline $\mathrm{P}$ & ${ }^{4} \mathrm{P}$ & -340.70966 & -340.80942 & -341.09335 & -341.11091 & -341.14332 & 3.8 & 3.8 \\
& ${ }^{4} \mathrm{P}$ & -340.47462 & -340.55066 & -340.84550 & -340.88789 & -340.90378 & 3.8 & 3.8 \\
\hline $\mathrm{S}$ & ${ }^{3} \mathrm{P}$ & -397.50269 & -397.64399 & -397.93148 & -397.94782 & -397.98359 & 2.0 & 2.0 \\
& ${ }^{3} \mathrm{~S}$ & -397.25466 & -397.38664 & -397.65653 & -397.69623 & -397.71533 & 2.3 & 2.4 \\
\hline $\mathrm{Cl}$ & ${ }^{2} \mathrm{P}$ & -459.47302 & -459.66785 & -459.94923 & -459.96961 & -460.00897 & 0.8 & 0.8 \\
& ${ }^{2} \mathrm{P}$ & -459.14429 & -459.31782 & -459.59664 & -459.64414 & -459.66317 & 0.9 & 1.0 \\
\hline $\mathrm{Ar}$ & ${ }^{1} \mathrm{~S}$ & -526.79442 & -527.04772 & -527.31586 & -527.34106 & -527.38515 & 0.0 & 0.0 \\
& ${ }^{1} \mathrm{P}$ & -526.38081 & -526.59620 & -526.88315 & -526.93537 & -526.95504 & 0.0 & 0.0 \\
\hline
\end{tabular}

${ }^{a} \mathrm{MC}-\mathrm{PDFT}$

${ }^{b}$ TD-KS-DFT

Table S9C. Total energies (in atomic units) for all states and $<\mathrm{S}^{2}>$ values with the d-aug-ccpVQZ basis set for Rydberg excitations of atoms $\mathrm{H}$ to $\mathrm{Ne}$.

\begin{tabular}{|c|c|c|c|c|c|c|c|c|}
\hline & states & CASSCF & CASPT2 & tPBE $^{a}$ & PBE $^{b}$ & PBE0 $^{b}$ & $\begin{array}{c}\text { PBE } \\
<\mathrm{S}^{2}>\end{array}$ & $\begin{array}{c}\text { PBE0 } \\
<\mathrm{S}^{2}>\end{array}$ \\
\hline $\mathrm{H}$ & ${ }^{2} \mathrm{~S}$ & -0.49995 & -0.49995 & -0.49937 & -0.49994 & -0.50127 & 0.8 & 0.8 \\
& ${ }^{2} \mathrm{~S}$ & -0.12490 & -0.12490 & -0.13588 & -0.21931 & -0.19111 & 0.8 & 0.8 \\
\hline $\mathrm{He}$ & ${ }^{1} \mathrm{~S}$ & -2.85199 & -2.89513 & -2.88053 & -2.89278 & -2.89502 & 0.0 & 0.0 \\
& ${ }^{1} \mathrm{~S}$ & -2.12137 & -2.14314 & -2.13449 & -2.29719 & -2.24440 & 0.0 & 0.0 \\
\hline $\mathrm{B}$ & ${ }^{2} \mathrm{P}$ & -24.55971 & -24.59431 & -24.60765 & -24.61121 & -24.61920 & 0.8 & 0.8 \\
& ${ }^{2} \mathrm{~S}$ & -24.39626 & -24.41495 & -24.42428 & -24.46960 & -24.45869 & 0.8 & 0.8 \\
\hline $\mathrm{C}$ & ${ }^{3} \mathrm{P}$ & -37.69951 & -37.77553 & -37.78438 & -37.79752 & -37.80662 & 2.0 & 2.0 \\
& ${ }^{3} \mathrm{P}$ & -37.45406 & -37.50525 & -37.51315 & -37.58561 & -37.56713 & 2.0 & 2.0 \\
\hline $\mathrm{N}$ & ${ }^{4} \mathrm{~S}$ & -54.37586 & -54.51296 & -54.49727 & -54.53421 & -54.54545 & 3.8 & 3.8 \\
& ${ }^{4} \mathrm{P}$ & -54.05065 & -54.13693 & -54.14746 & -54.24035 & -54.21481 & 3.8 & 3.8 \\
\hline $\mathrm{O}$ & ${ }^{3} \mathrm{P}$ & -74.80389 & -74.97925 & -74.98770 & -75.01286 & -75.02117 & 2.0 & 2.0 \\
& ${ }^{3} \mathrm{~S}$ & -74.46216 & -74.64659 & -74.60381 & -74.73405 & -74.70937 & 3.2 & 3.2 \\
\hline $\mathrm{F}$ & ${ }^{2} \mathrm{P}$ & -99.38309 & -99.63580 & -99.63017 & -99.67311 & -99.67311 & 0.8 & 0.8 \\
& ${ }^{2} \mathrm{P}$ & -98.95708 & -99.17136 & -99.15607 & -99.29431 & -99.25112 & 2.0 & 2.0 \\
\hline $\mathrm{Ne}$ & ${ }^{1} \mathrm{~S}$ & -128.47625 & -128.83339 & -128.83309 & -128.86234 & -128.86804 & 0.0 & 0.0 \\
& ${ }^{1} \mathrm{P}$ & -127.97361 & -128.22213 & -128.23601 & -128.36518 & -128.31655 & 0.0 & 0.0 \\
\hline
\end{tabular}

${ }^{a} \mathrm{MC}-\mathrm{PDFT}$

${ }^{b}$ TD-KS-DFT 
Table S10C. Total energies (in atomic units) for all states and $<\mathrm{S}^{2}>$ values with the aug-cc-pV5Z basis set for Rydberg excitations of atoms $\mathrm{H}$ to $\mathrm{Ne}$.

\begin{tabular}{|c|c|c|c|c|c|c|c|c|}
\hline & states & CASSCF & CASPT2 & tPBE $^{a}$ & $\mathrm{PBE}^{b}$ & PBE0 $^{b}$ & $\begin{array}{c}\text { PBE } \\
<\mathrm{S}^{2}>\end{array}$ & $\begin{array}{c}\text { PBE0 } \\
<\mathrm{S}^{2}>\end{array}$ \\
\hline $\mathrm{H}$ & ${ }^{2} \mathrm{~S}$ & -0.49999 & -0.49999 & -0.49941 & -0.49998 & -0.50132 & 0.8 & 0.8 \\
& ${ }^{2} \mathrm{~S}$ & -0.12482 & -0.12482 & -0.13595 & -0.20843 & -0.18554 & 0.8 & 0.8 \\
\hline $\mathrm{He}$ & ${ }^{1} \mathrm{~S}$ & -2.85291 & -2.89633 & -2.88198 & -2.89288 & -2.89512 & 0.0 & 0.0 \\
& ${ }^{1} \mathrm{~S}$ & -2.11429 & -2.13581 & -2.13242 & -2.25108 & -2.21056 & 0.0 & 0.0 \\
\hline $\mathrm{B}$ & ${ }^{2} \mathrm{P}$ & -24.55994 & -24.59537 & -24.60873 & -24.61198 & -24.61967 & 0.8 & 0.8 \\
& ${ }^{2} \mathrm{~S}$ & -24.39478 & -24.41392 & -24.42377 & -24.46137 & -24.45333 & 0.8 & 0.8 \\
\hline $\mathrm{C}$ & ${ }^{3} \mathrm{P}$ & -37.70053 & -37.77768 & -37.78363 & -37.79844 & -37.80726 & 2.0 & 2.0 \\
& ${ }^{3} \mathrm{P}$ & -37.44916 & -37.50209 & -37.51011 & -37.57061 & -37.55630 & 2.0 & 2.0 \\
\hline $\mathrm{N}$ & ${ }^{4} \mathrm{~S}$ & -54.38002 & -54.51606 & -54.50051 & -54.53539 & -54.54636 & 3.8 & 3.8 \\
& ${ }^{4} \mathrm{P}$ & -54.04136 & -54.13202 & -54.14215 & -54.21850 & -54.19863 & 3.8 & 3.8 \\
\hline $\mathrm{O}$ & ${ }^{3} \mathrm{P}$ & -74.80566 & -74.98615 & -74.98720 & -75.01456 & -75.01456 & 2.0 & 2.0 \\
& ${ }^{3} \mathrm{~S}$ & -74.44205 & -74.63038 & -74.59048 & -74.69390 & -75.02263 & 3.4 & 3.4 \\
\hline $\mathrm{F}$ & ${ }^{2} \mathrm{P}$ & -99.38680 & -99.64650 & -99.62749 & -99.67555 & -99.68156 & 0.8 & 0.8 \\
& ${ }^{2} \mathrm{P}$ & -98.92778 & -99.15063 & -99.13339 & -99.22242 & -99.18926 & 2.0 & 1.2 \\
\hline $\mathrm{Ne}$ & ${ }^{1} \mathrm{~S}$ & -128.48396 & -128.84806 & -128.78269 & -128.86573 & -128.87123 & 0.0 & 0.0 \\
& ${ }^{1} \mathrm{P}$ & -127.93535 & -128.19679 & -128.19941 & -128.30330 & -128.26393 & 0.0 & 0.0 \\
\hline
\end{tabular}

${ }^{a} \mathrm{MC}-\mathrm{PDFT}$

${ }^{b}$ TD-KS-DFT

Table S11C. Total energies (in atomic units) for all states and $<\mathrm{S}^{2}>$ values with the d-aug-ccpV5Z basis set for Rydberg excitations of atoms $\mathrm{H}$ to $\mathrm{Ne}$.

\begin{tabular}{|c|c|c|c|c|c|c|c|c|}
\hline & states & CASSCF & CASPT2 & tPBE $^{a}$ & PBE $^{b}$ & PBE0 $^{b}$ & $\begin{array}{c}\text { PBE } \\
<\mathrm{S}^{2}>\end{array}$ & $\begin{array}{c}\text { PBE0 } \\
<\mathrm{S}^{2}>\end{array}$ \\
\hline $\mathrm{H}$ & ${ }^{2} \mathrm{~S}$ & -0.49999 & -0.49999 & -0.49941 & -0.49998 & -0.50132 & 0.8 & 0.8 \\
& ${ }^{2} \mathrm{~S}$ & -0.12494 & -0.12494 & -0.13579 & -0.21949 & -0.19105 & 0.8 & 0.8 \\
\hline $\mathrm{He}$ & ${ }^{1} \mathrm{~S}$ & -2.85207 & -2.89612 & -2.88057 & -2.89288 & -2.89512 & 0.0 & 0.0 \\
& ${ }^{1} \mathrm{~S}$ & -2.12174 & -2.14360 & -2.13467 & -2.29775 & -2.24468 & 0.0 & 0.0 \\
\hline $\mathrm{B}$ & ${ }^{2} \mathrm{P}$ & -24.55986 & -24.59541 & -24.60764 & -24.61198 & -24.61967 & 0.8 & 0.8 \\
& ${ }^{2} \mathrm{~S}$ & -24.39637 & -24.41535 & -24.42437 & -24.47021 & -24.45906 & 0.8 & 0.8 \\
\hline $\mathrm{C}$ & ${ }^{3} \mathrm{P}$ & -37.69984 & -37.77780 & -37.78363 & -37.79845 & -37.80726 & 2.0 & 2.0 \\
& ${ }^{3} \mathrm{P}$ & -37.45434 & -37.50673 & -37.51011 & -37.58656 & -37.56782 & 2.0 & 2.0 \\
\hline $\mathrm{N}$ & ${ }^{4} \mathrm{~S}$ & -54.37649 & -54.51674 & -54.49786 & -54.53539 & -54.54636 & 3.8 & 3.8 \\
& ${ }^{4} \mathrm{P}$ & -54.05126 & -54.13986 & -54.14860 & -54.24172 & -54.21590 & 3.8 & 3.8 \\
\hline $\mathrm{O}$ & ${ }^{3} \mathrm{P}$ & -74.80507 & -74.98615 & -75.01612 & -75.01456 & -75.02263 & 2.0 & 2.0 \\
& ${ }^{3} \mathrm{~S}$ & -74.46340 & -74.65118 & -74.60013 & -74.73632 & -74.71126 & 3.2 & 3.2 \\
\hline $\mathrm{F}$ & ${ }^{2} \mathrm{P}$ & -99.38509 & -99.64642 & -99.62560 & -99.67556 & -99.68156 & 0.8 & 0.8 \\
& ${ }^{2} \mathrm{P}$ & -98.95912 & -99.17956 & -99.15613 & -99.29773 & -99.26032 & 2.0 & 2.0 \\
\hline $\mathrm{Ne}$ & ${ }^{1} \mathrm{~S}$ & -128.47932 & -128.84793 & -128.82079 & -128.86574 & -128.87123 & 0.0 & 0.0 \\
& ${ }^{1} \mathrm{P}$ & -127.97682 & -128.23429 & -128.23190 & -128.37077 & -128.32146 & 0.0 & 0.0 \\
\hline
\end{tabular}

${ }^{a} \mathrm{MC}-\mathrm{PDFT}$

${ }^{b}$ TD-KS-DFT 
S-13

${ }^{\mathrm{S} 1}$ http://physics.nist.gov/PhysRefData/ASD/Levels_form.html 